\title{
Giant Magnetic Effects and Oscillations in Antiferromagnetic Josephson Weak Links
}

\author{
L. Gor'kov a,b and V. Kresinc \\ (a) National High Magnetic Field Laboratory, Florida State \\ University, Tallahassee, Fl 32310 \\ (b) L. D. Landau Institute for Theoretical Physics, Russian \\ Academy of Sciences, 117334 Moscow, Russia \\ (c) Lawrence Berkeley Laboratory, University of California, Berkeley, Ca 94720
}

\begin{abstract}
$\underline{\text { Abstract }}$
Josephson junctions with an antiferromagnetic metal as a link are described. An especially interesting case is provided by a barrier built on a giant magnetoresistance (GMR) multilayer or by using doped manganites in the metallic A-phase. Such a junction is predicted to display unusual properties. The junction can be switched off by a relatively small magnetic field. In addition, the amplitude of the Josephson current rapidly oscillates as a function of the field.
\end{abstract}


Studies of magnetic systems, such as giant magnetoresistance (GMR) structures (see, e.g. [1,2]), mixed-valence manganites [3], magnetic dilute semiconductors, etc. have resulted in invention of many new devices. In this paper we describe two phenomena for magnetic systems such as GMR layered structures incorporated into a superconducting tunneling junction. Combination of superconductivity and magnetism in electronics turns out to be promising from the viewpoint of interesting applications.

In addition to common realization of Josephson contacts (i.e., S-I-S or S-N-S junctions with an insulating (I), or metallic (N) weak link connecting two superconductors), we consider a magnetic metallic barrier. For two superconductors with the singlet pairing (s or d-wave) a ferromagnetic barrier would present a strong obstacle (in the absence of the spin-orbital coupling), because of the pair breaking effect of the exchange field on a Cooper pair

In this paper we explore the Josephson contact built up of an array of ferromagnetic layers with alternating directions of magnetization. To the best of our knowledge, antiferromagnetic metallic weak links have never been discussed in any detail. Here we consider the specific situation of a GMR heterostructure with conducting layers ( spin- valve effect). The Josephson currents flow along ferromagnetic layers. Layers are weakly coupled electronically. Heterostructures of this kind are common in various GMR applications and their fabrication by now is a well elaborated process $[1,2]$ . It is appropriate to mention here that such a structure realizes itself on the microscopic level as the so-called A-phase in doped manganites.

It is shown theoretically in the next section that such S-AFM-S system results in a junction, where the amplitude of the Josephson current displays two remarkable features: (1) current can be switched off by a relatively small external magnetic field, and 
(2) the amplitude shows rapid oscillations as a function of the applied field. These oscillations are originated from gradual weak canting of the magnetic moments in the presence of applied field.

Consider the Josephson junction with two superconducting electrodes, $\mathrm{S}_{1}, \mathrm{~S}_{2}$ connected by a magnetic metallic weak link formed by a multilayer structure described above . Antiferromagnetic (AFM) ordering runs along the c-direction perpendicular to the layers (A-phase).

We keep in mind the possibility of the two realizations. The first one is an artificial (GMR) heterostructure of alternating ferromagnetic and non-magnetic layers. The non-magnetic layer can be either an insulator (e.g. $\mathrm{Co}_{-} \mathrm{Al}_{2} \mathrm{O}_{3}-\mathrm{Co}-\ldots$ system [4]) or non-magnetic metal. The AFM alignment is supposed for the adjacent ferromagnetic layers .

Another exciting possibility is provided by the recently discovered metallic Aphase in doped manganites. For example, doped compounds $\mathrm{A} 1-\mathrm{x} \mathrm{Sr}_{\mathrm{X}} \mathrm{MnO}_{3}$ ( $\mathrm{A}=\mathrm{La}, \mathrm{Nd}$ ) with $\mathrm{x}=0.55$ or $\mathrm{Pr}_{0.5} \mathrm{Sr}_{0.5} \mathrm{MnO}_{3}$ belong to this category [5-7]. Their conductivity displays a strong anisotropic behavior. The magnetic structure corresponds to ferromagnetic atomic layers with the AFM ordering along one of the cubic axis (see the review [3]). The system is thus quite similar to artificial GMR superlattices, although the physics is mainly governed by the strong Hund interaction which is the source of the double exchange (DE) mechanism for the ferromagnetism in manganites [8]. Concurrently, this mechanism switches off coupling between antiferromagnetically aligned adjacent layers in the A-phase. Note that ferromagnetism in manganites corresponds to the so-called " half-metallic" state, i.e. , to the complete polarization of carriers.

To simplify the treatment below we assume that the distance between the superconductors, $\mathrm{L}$, is much larger than the proximity coherence length inside the connecting barrier, so that $\mathrm{L}>>\zeta_{\mathrm{N}}$; 
$\zeta_{\mathrm{N}}=\mathrm{hv}_{\mathrm{F}} / 2 \pi \mathrm{T}$. This allows one to use an effective interface Hamiltonian between a superconductor and a metal in the form:

$$
\mathrm{H}_{\text {pair }}=\mathrm{V} \Delta_{\mathrm{i}} \Psi^{+}(\mathrm{i}) \Psi^{+}(\mathrm{i})
$$

where $\mathrm{V}$ is a matrix element for "conversion" of the pair condensate, $\Delta_{\mathrm{i}}$, for the i-th superconductor into two electrons at the metallic interface; $\Psi(\mathrm{i})$ and $\Psi^{+}(\mathrm{i})$ are the field operators for electrons of the barrier. With the use of Eq.(1) we find a correction to the thermodynamic potential, $\delta \Omega$, caused by the barrier; the current is obtained as $\delta \Omega / \delta \varphi$ with $\varphi$ being a phase difference between two superconductors' order parameters. As a result, the problem reduces to calculating the Cooper term (see, e.g., [9]) :

$\mathrm{K}=\pi \mathrm{T}\left|\mathrm{V}^{2}\right| \sum_{\omega_{\mathrm{n}}} \Delta_{1} \Delta_{2} \int \mathrm{d} \mathbf{p d} \mathbf{q} \exp (\mathbf{i q} \mathbf{L}) \Pi\left(\mathbf{q}, \mathrm{i} \omega_{\mathrm{n}}\right)$

where

$$
\begin{aligned}
& \Pi\left(i \omega_{n}, \mathbf{q}\right)=\sum_{\sigma, \sigma, \sigma^{\prime \prime, \sigma^{\prime}}}\left(\hat{\sigma}_{y}\right)_{\sigma, \sigma^{\prime}} G_{\sigma, \sigma^{\prime}}\left(i \omega_{n}, \mathbf{p}\right)\left(\hat{\sigma}_{y}\right)_{\sigma^{\prime \prime}, \sigma^{\prime \prime}} \\
& * G_{\sigma^{\prime}, \sigma^{\prime \prime}}\left(-i \omega_{n}, \mathbf{q}-\mathbf{p}\right)= \\
& 2\left[G_{\downarrow \uparrow}\left(i \omega_{n}, \mathbf{p}\right) G_{\uparrow \downarrow}\left(-i \omega_{n}, \mathbf{q}-\mathbf{p}\right)-G_{\downarrow \downarrow}\left(i \omega_{n}, \mathbf{p}\right) G_{\uparrow \uparrow}\left(-i \omega_{n}, \mathbf{q}-\mathbf{p}\right)\right]
\end{aligned}
$$

In the above $\Delta_{1}, \Delta_{2}$ are the order parameters for two superconductors $\left(\Delta_{\mathrm{i}}=\left|\Delta_{\mathrm{i}}\right| \exp \left(\mathrm{i} \varphi_{\mathrm{i}}\right), \omega_{\mathrm{n}}=(2 \mathrm{n}+1) \pi \mathrm{T}\right.$ (we use the method of thermodynamic Green' functions, see e.g. [9]), $L$ is the distance from $S_{1}$ to $S_{2}$.

For concretness we perform the calculation for the A-phase in manganites. We show below that the results have quite a general character and are also applicable for AFM GMR multilayers Consider a general case of the canted magnetic structure. The Hamiltonian has the form (cf.[10]): 


$$
\begin{aligned}
& \mathrm{H}=\sum_{\mathbf{p}} \mathrm{t}(\mathbf{p}) \mathrm{a}_{\mathbf{p} \sigma}^{+} \mathrm{a}_{\mathbf{p} \sigma \sigma^{+}}^{+}+\sum_{\mathbf{p}, \mathbf{Q}} \mathrm{J}_{\mathrm{H}} \mathrm{S}(\mathbf{Q}) \mathrm{a}_{\mathbf{p} \sigma^{+}}^{+}\left(\hat{\sigma}_{z}\right)_{\sigma^{\prime \prime} \sigma^{\prime}} \mathbf{a}_{\mathbf{p}-\mathbf{Q} ; \sigma^{\prime \prime}} \\
& +\sum_{\mathbf{p}, \mathbf{Q}} \mathrm{J}_{\mathrm{H}} \mathrm{S}(-\mathbf{Q}) \mathrm{a}_{\mathbf{p} \sigma^{+}}^{+}\left(\hat{\sigma}_{z}\right)_{\sigma^{\prime \prime} \sigma^{\prime \prime}} \mathrm{a}_{\mathbf{p}-\mathbf{Q} ; \sigma^{\prime \prime}}+\sum_{\mathbf{p}} \mathrm{J}_{\mathrm{H}} \mathrm{Ma}_{\mathbf{p} \sigma^{+}}^{+}\left(\hat{\sigma}_{\mathrm{x}}\right)_{\sigma^{\prime} \sigma^{\prime \prime}} \mathrm{a}_{\mathbf{p}-\mathbf{Q} ; \sigma^{\prime \prime}}
\end{aligned}
$$

Here in $\mathrm{t}=\mathrm{t}_{\perp}+\mathrm{t}_{||}, \mathrm{t}_{\perp}$ and $\mathrm{t}_{||}$are electron hopping parameters for the in-plane and out-of plane motion, $\mathrm{J}_{\mathrm{H}}$ is the Hund's coupling, and $\mathrm{S}(\mathbf{Q})$ is the Fourier component of the AFM ordering along the c-directions; $<\mathrm{S}_{\mathrm{Z}^{>}}=\mathrm{S}(-1)^{\mathrm{n}}$. The structural vector $\mathbf{Q}=(0,0, \pi / \mathrm{a})$ reduces the Brillouin zone ( $\mathrm{a}$ is the lattice constant); $\mathrm{M}$ is the canted magnetic moment: $S_{i}=\left( \pm<S_{z}>, M_{x}\right), S_{z}^{2}+M_{x}^{2}=S(S+1) \cong S^{2}$. Orientations of $M$ and $S$ are fixed by a magnetic anisotropy and the external field.

Using equation of motion:

$$
\left(\tilde{\varepsilon}-t_{11}\right) a_{k \sigma}=J_{H} S( \pm Q) \hat{\sigma}_{z} a_{k-Q ; \sigma}+J_{H} M\left(\hat{\sigma}_{x}\right)_{\sigma \sigma^{\prime}} a_{k \sigma^{\prime}}
$$

and similar equation for $\mathbf{k}-->\mathbf{k}+\mathbf{Q}$, where $\tilde{\varepsilon}=\varepsilon-\mathrm{t}_{\perp}$, one can diagonalize the Hamiltonian (3). The energy spectrum is:

$\tilde{\varepsilon}= \pm\left[\mathrm{J}_{\mathrm{H}}^{2} \mathrm{~S}^{2} \pm 2 \mathrm{~J}_{\mathrm{H}} \mathrm{Mt}_{11}+\mathrm{t}_{1 \mathrm{I}}^{2}\right]^{-2}$

Remember that DE mechanism for manganites [8] exploits the large value of the Hund interaction, $\mathrm{J}_{\mathrm{H}} \approx 1 \mathrm{eV}[10]$, so that $\mathrm{J}_{\mathrm{H}}>>$ t. Therefore electrons occupy only the two lowest bands:

$$
\tilde{\varepsilon}_{1,2} \cong-\mathrm{J}_{\mathrm{H}} \mathrm{S} \pm(\mathrm{M} / \mathrm{S}) \mathrm{t}_{\mathrm{II}}
$$


By performing the canonical transformation in accordance with the eigenvalues of Eq. (5), it is a straightforward to express operators $\hat{\Psi}$ and $\hat{\Psi}^{+}$in Eq.(1) in terms of the new eigenfunctions. Quite generally, the transformation has the form:

$$
\mathrm{a}_{\mathbf{p \sigma}}=\sum_{\mathrm{i}} \mathrm{K}_{\sigma \mathrm{i}} \alpha_{\mathrm{ip}}
$$

where $\sigma \equiv(\uparrow \downarrow), \mathrm{K}_{\sigma 1}=0.5[1-(\mathrm{M} / \mathrm{S})]^{1 / 2} ; \mathrm{K}_{\uparrow 2}=-\mathrm{K}_{\downarrow}=0.5[1+(\mathrm{M} / \mathrm{S})]^{1 / 2}$

With the use of Eqs. (2), (5), (6), we calculate the amplitude of the Josephson current, to obtain:

$$
\mathrm{j}_{\mathrm{m}}=A e^{\frac{\mathrm{L}}{\xi_{\mathrm{N}}(\mathrm{T})}} \mathrm{J}_{0}(\beta \mathrm{M} / \mathrm{S})
$$

Here $\xi_{\mathrm{N}}$ is the coherence length inside the barrier (see above),

$\mathrm{A}=\left[1-(\mathrm{M} / \mathrm{S})^{2}\right] \gamma, \beta=\left(\mathrm{t}_{0} / \mathrm{T}_{\mathrm{c}}\right)\left(\mathrm{L} / \xi_{0}\right) ; \xi_{0}=\mathrm{hv}_{\mathrm{F}} / 2 \pi \mathrm{T}_{\mathrm{c}}$

In the tight-binding approximation $\mathrm{t}_{1 \mid}=\mathrm{t}_{\mathrm{O}} \cos \left(\mathrm{p}_{\mathrm{Z}} \mathrm{d}_{\mathrm{c}}\right)$; we will not write out the explicit expression for the coefficient $\gamma \propto|V|^{2}$. Note that in Eq.(7) $\beta>>1$, since either $\mathrm{t}_{0}>>\mathrm{Tc}$ (for manganites, see [10]), or $\mathrm{L}>>\xi_{0}$ (see above). If $\mathrm{M} / \mathrm{S}$ is not exceedingly small, the Bessel function $\mathrm{J}_{0}(\mathrm{x})$ in (7) may be written in the asymptotic form: $\mathrm{J}_{0}(\beta \mathrm{M} / \mathrm{S}) \approx(\pi \beta \mathrm{M} / 2 \mathrm{~S})^{-1 / 2} \cos (\beta \mathrm{M} / \mathrm{S}-\pi / 4)$.

Before discussing the expression (7), let us make several comments regarding minor changes needed to make it applicable to the Josephson current through a GMR structure. Generally speaking, GMR effects are sensitive to the number of the M-NM bilayers, $\mathrm{n}$ (M and NM are magnetic and non-magnetic layers, correspondingly). GMR films with $\mathrm{n}$ up to $\mathrm{n}=50$ are currently available. A saturation is already set in for $\mathrm{n}>10$ [11], see also the review [1]. Therefore, multilayer GMR structures with $n>10$ represents a "bulk anisotropic crystal" quite similar to the A-phase in manganites. The new structural vector $\mathbf{Q}$ would be $\mathbf{Q}=(0,0, \pi / \mathrm{d})$ where $\mathrm{d}$ is the thickness of single bilayer. 
In the Hamiltonian (3) $\mathrm{J}_{\mathrm{H}}$ must be replaced by $\mathrm{J}$, the exchange coupling, and $\mathrm{S}$ corresponds to magnetization of the ferromagnetic layer. It is also clear that the anisotropy in tunneling gets stronger: $\mathrm{t}_{1 \mathrm{I}}<<\mathrm{t}$ and the exchange could be comparable with the bandwidth, $t$. As a result, all four branches in the spectrum (5) contribute into the current. In other respects, the calculation run in parallel leading to the same expression (7); as for the parameters, they are different, first of all because for the GMR multilayers, unlike manganites, the out-of plane hopping can be rather weak, especially for systems with insulating $\mathrm{Al}_{2} \mathrm{O}_{3}$ layers. Note, however, that the inequality $\mathrm{L}>>\xi_{0}$ still holds.

The multilayer system consisting of alternating $\mathrm{Co}$ and $\mathrm{Al}_{2} \mathrm{O}_{3}$ layers is an example of such system. As a magnetic layers, one can use also the NiCo compound. Realistic parameters for such GMR barrier are $: \mathrm{d}_{\mathrm{Co}} ; \mathrm{d}_{\mathrm{NiC}_{0}} \approx 30 \mathrm{~A}, \mathrm{~d}_{\mathrm{Al}_{2} \mathrm{O}_{3}} \approx 6 \mathrm{~A}, \mathrm{t}_{0} \approx 1-5 \mathrm{~K}, \mathrm{~L} \approx$ $10^{3} \mathrm{~A}[4]$.

Equation (7) is the main result of the paper. One sees that the antiferromagnetic barrier in accordance with Eq. (7) transfers the Josephson current; in this case the exchange field does not break the Cooper pair. There is the oscillating factor in the current amplitude $\mathrm{J}_{0}(\beta \mathrm{M} / \mathrm{S}) \propto \operatorname{Cos}(\beta \mathrm{M} / \mathrm{S})$ which results in rapid oscillations in the current through the barrier as the function of magnetization $M$. Since $\beta>>1$, a small $M$ induced by an external field, leads to a giant impact on the Josephson current. It is remarkable that even small deviations from the exact AFM ordering lead to giant oscillations of the amplitude of the current which passes through zero at $\mathrm{J}_{0}(\beta \mathrm{M} / \mathrm{S})=0$. At the further increase of the external field the AFM-->FM transition takes place as usual for the GNR effect (see e.g. [1,2]). For the GMR films, the low operating fields(order of 3-4 Oe [4] ) allow to cut-off the junction in accordance with expression ( $\left.7^{\prime}\right)$ for the amplitude $\mathrm{A}$ in $(7)$. Manganites would require higher fields $(\approx 5-10 \mathrm{~T})$. 
Of much greater interest is the oscillatory dependence of the amplitude of the Josephson current on an applied magnetic field.

We dubbed this as a Giant Magnetooscillations for the Josephson contact with the Astructure.

Note that this effect is entirely different from usual dampened Fraunhofer oscillations which occur due to the field penetrating into the barrier. In our case the field direction can be such that only orientation of magnetic moments in the barrier is affected.

To conclude, we suggest experiments with the S - AFM - S junction to verify the picture described above. The use of ordinary superconductors, such as $\mathrm{Nb}$, would be a natural choice.

The authors thank S.S.P. Parkin for interesting and fruitful discussions. The work was supported(L.P.G.) by the National High Magnetic Field Laboratory through the NSF cooperative agreement No. DMR-9521035 and The State of Florida, and the Naval Research Laboratory Grant N00173-00-1-6005, and (V. Z. K.) by DARPA under the Contract No. 01J543. 


\section{References.}

1. S.S.P.Parkin, Giant Magnetoresistance in Magnetic Nanostructures, in Ann. Rev. Mater. Sci. 25, 358 (1995).

2. Ultrathin Magnetic Structures II, B.Heinrich and

J.A.C.Bland, Eds., Springer-Verlag,Berlin (1994).

3. M.D.Coey, M.Viret, and S. von Molnar, Adv. Phys. 48, 167 (1999).

4. S.S.P.Parkin, preprint (2001).

5. H.Kawano, R.Kajimoro, H.Yoshizawa, Y.Tomioka, H.Kuwahara, Y.Tokura, Phys.Rev.Lett. $\underline{78}, 4253$ (1997); H. Fujisturo,M.Ikebe,

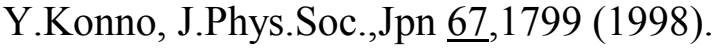

6. M.Isumi,T.Manako,Y.Konishi, M.Kawasaki,Y.Tokura, Phys.Rev.B61,12187(2000); H.Kuwakara, T.Okuda, Y.Tomioko, A.Asamitsu, Phys.Rev.Lett르,4316 (1999); R.Kajimoto, H.Yoshizawa, H.Kawano, H.Kuwahara, Y.Tokura, K.Ohoyama, M.Ohashi, Phys.Rev.B무, 9506 (1999).

7. T.Akimoto,Y.Maruyama, Y.Morimoto, A.Nakamura, K.Hirota, K.Ohoyama, M.Ohashi, Phys.Rev.B6ㅜ, R5594 (1998); Y.Morimoto, T.Akimoto, A.Nakamura, K.Ohoyama, M.Ohashi, Phys.Rev.B토,5544 (1998).

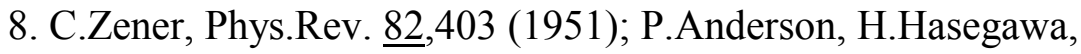
Phys.Rev.100,675 (1955).

9. A.Abrikosov, L.Gor'kov,I.Dzyaloshinskii, Methods of Quantum Field Theory in Statistical Physics, Dover, NY (1975).

10. L.Gor'kov and V.Kresin, JETP Lett. 67, 985 (1998); M.Dzero, L.Gor'kov, and V.Kresin, Eur. Phys. J.B 14,459 (2000).

11. E.Fullerton, M.Conover, J.Mattson, C.Sowers, S.Bader, Appl.Phys. 
Lett., $\underline{63}, 1698$ (1993); R.Schad, C.Potter, P.Belien, G.Verbanck, V.Moshchalkov, Y.Brueseraede, Appl.Phys.Lett. 64,3500(1994) 
